\title{
Note on ingoing coordinates for binary black holes
}

\author{
Kashif Alvi \\ Theoretical Astrophysics, California Institute of Technology, Pasadena, California 91125
}

(Received 13 February 2003; published 9 May 2003)

\begin{abstract}
In a previous paper, a binary black hole four-metric was presented in a post-Newtonian corotating coordinate system valid only up to the holes' apparent horizons. In this paper, I define an ingoing coordinate transformation that extends this corotating coordinate system through the holes' horizons and into their interiors. The motivation for using ingoing coordinates is that numerical simulations of black holes require the computational grid to extend inside the horizons. The coordinate transformation presented here makes the binary black hole four-metric suitable as a source of initial data for numerical simulations.
\end{abstract}

DOI: 10.1103/PhysRevD.67.104006

PACS number(s): 04.25.Dm, 04.25.Nx, 04.30.Db

\section{INTRODUCTION}

In a previous paper [1], an approximate solution to Einstein's equations representing two widely separated nonrotating black holes in a circular orbit was constructed by matching a post-Newtonian metric to two perturbed Schwarzschild metrics. The spacetime metric was presented in a single coordinate system valid up to the holes' apparent horizons. In this paper, I write the binary black hole four-metric from [1] in coordinates that are corotating and post-Newtonian in the radiation and near zones and smoothly become ingoing near the black holes. This coordinate system is valid through the holes' horizons and covers the holes' interiors as well as the near and radiation zones. The metric components in this coordinate system are explicitly nonsingular on the black hole horizons. The metric presented here is promising as a source of initial data for numerical simulations of binary black holes. Since these simulations require the computational grid to extend inside the holes' horizons, the coordinate system used near the black holes in [1] is not suitable for numerical relativity; in that coordinate system, the metric components diverge at the holes' horizons. For further discussion of the suitability of various coordinate systems for numerical relativity, see [2] and references therein.

Let us begin with the metric near the first black hole, that is, in region I in the terminology of [1] (see Fig. 1 in [1]). This metric is the Schwarzschild metric plus electric-type and magnetic-type tidal perturbations due to the second black hole, and is given in isotropic coordinates in Eq. (3.22) of [1]. The second black hole's tidal field rotates with angular velocity $\Omega$ as seen by inertial observers in the first black hole's local asymptotic rest frame. However, the tidal perturbation's angular dependence $\phi-\Omega \tilde{t}$ as given in Eq. (3.22) of [1] (I have replaced $T$ in that equation with $\tilde{t}$ ) is singular at the first black hole's horizon. The reason is that the Schwarzschild time coordinate $\tilde{t}$, which is suitable for applying the technique of matched asymptotic expansions in the buffer zone around the black hole (see [1] for details), is badly behaved at the horizon. Since our goal in this paper is to come up with coordinates valid through the horizon and inside the black hole, we must use a time coordinate $T$ with the property that the hypersurfaces of constant time coincide with Schwarzschild time slices in the buffer zone but smoothly transition into ingoing Eddington-Finkelstein time slices which penetrate the horizon. The singular angular dependence $\phi-\Omega \tilde{t}$ can be simply replaced by the nonsingular $\phi-\Omega T$, with $T$ as described above; this is discussed in further detail below.

It turns out that isotropic coordinates are not a good starting point for an ingoing transformation. The analog of the ingoing Eddington-Finkelstein transformation, which is based on ingoing null geodesics of the Schwarzschild spacetime, is unsuccessful when applied to isotropic coordinates: the coordinate system remains singular at the horizon. Indeed, the isotropic radial coordinate is only defined outside the black hole. However, isotropic coordinates were used in [1] to match a tidally perturbed Schwarzschild metric to the post-Newtonian near zone metric. It is therefore necessary to define a new radial coordinate that is equal to the (tidally distorted) isotropic radial coordinate in the buffer zone but transitions smoothly into the (tidally distorted) Schwarzschild radial coordinate near the black hole.

\section{INGOING COORDINATES}

Following the notation in [1], I denote the black holes' masses by $m_{1}$ and $m_{2}$, and their coordinate separation in post-Newtonian harmonic coordinates by $b$. Let $m=m_{1}$ $+m_{2}, \epsilon=(m / b)^{1 / 2}$, and $\Omega=\left(1-m_{1} m_{2} / m b\right)\left(m / b^{3}\right)^{1 / 2}$. By assumption, $\epsilon \ll 1$.

Let us begin with the region I metric given in isotropic coordinates in Eq. (3.22) of [1]. Note that, in this paper, $T$ and $R$ denote the nonsingular time and radial coordinates described in Sec. I, while in [1], they denoted the isotropic time and radial coordinates. Set $\Omega=0$ in Eq. (3.22) of [1] and transform to Schwarzschild coordinates $(\tilde{t}, \tilde{r}, \theta, \phi)$. This yields the metric $\tilde{\mathbf{g}}=\mathbf{g}_{S}+\widetilde{\mathbf{h}}$; the Schwarzschild metric $\mathbf{g}_{S}$ and the stationary tidal perturbation $\widetilde{\mathbf{h}}$ are given in Schwarzschild coordinates by

$$
\begin{aligned}
\mathbf{g}_{S}= & -\left(1-\frac{2 m_{1}}{\tilde{r}}\right) d \tilde{t}^{2}+\left(1-\frac{2 m_{1}}{\tilde{r}}\right)^{-1} d \tilde{r}^{2} \\
& +\tilde{r}^{2}\left(d \theta^{2}+\sin ^{2} \theta d \phi^{2}\right),
\end{aligned}
$$




$$
\begin{aligned}
\widetilde{\mathbf{h}}= & -\frac{4 m_{2}}{b^{3}} \sqrt{\frac{m}{b}}\left(1-\frac{2 m_{1}}{\tilde{r}}\right) \tilde{r}^{3} d t[\cos \theta \sin \phi d \theta \\
& +\sin \theta \cos (2 \theta) \cos \phi d \phi] \\
& +\frac{m_{2} \tilde{r}^{2}}{b^{3}}\left[3 \sin ^{2} \theta \cos ^{2} \phi-1\right] \\
& \times\left[\left(1-\frac{2 m_{1}}{\tilde{r}}\right)^{2} d t^{2}+d \widetilde{r}^{2}\right. \\
& \left.+\left(\tilde{r}^{2}-2 m_{1}^{2}\right)\left(d \theta^{2}+\sin ^{2} \theta d \phi^{2}\right)\right] .
\end{aligned}
$$

In this notation, $d \tilde{t}, d \tilde{r}, d \theta$, and $d \phi$ are coordinate oneforms and $d \tilde{t}^{2}$ denotes the tensor product $d \tilde{t} \otimes d \tilde{t}$.

Let $\zeta_{1}$ and $\zeta_{2}$ be two numbers satisfying $2<\zeta_{1}<\zeta_{2}$ $<\left(b / m_{1}\right)^{1 / 2}$. Define the new ingoing coordinates $(T, R, \theta, \phi)$ by

$$
\begin{aligned}
& \tilde{t}=T-2 m_{1} \ln \left(\frac{R}{2 m_{1}}-1\right) \psi(R), \\
& \tilde{r}=R+m_{1}\left(1+\frac{m_{1}}{4 R}\right) \eta(R) .
\end{aligned}
$$

The functions $\psi(R)$ and $\eta(R)$ must satisfy the following properties: (i) for $R \geqslant \zeta_{2} m_{1}, \psi(R)=0$ and $\eta(R)=1$ so the coordinates are isotropic; (ii) for $R \leqslant \zeta_{1} m_{1}, \psi(R)=1$ and $\eta(R)=0$ so the time coordinate is Eddington-Finkelstein and the radial coordinate is Schwarzschild; and (iii) for $\zeta_{1} m_{1}<R<\zeta_{2} m_{1}, \psi(R)$ and $\eta(R)$ smoothly and monotonically vary between their constant values outside this interval. The transition points $R=\zeta_{1} m_{1}$ and $R=\zeta_{2} m_{1}$ and the functions $\psi(R)$ and $\eta(R)$ can be chosen freely as long as the above properties are satisfied. Since $d \tilde{r} / d R=1+\left(m_{1}\right.$ $\left.+m_{1}^{2} / 4 R\right) \eta^{\prime}(R)-m_{1}^{2} \eta(R) / 4 R^{2} \geqslant 1-m_{1}^{2} / 4 R^{2}$, we have $d \tilde{r} / d R>0$ for $R>2 m_{1}$, and so Eqs. (3) and (4) define a valid coordinate transformation.

Transforming (1) and (2) using (3) and (4), we obtain

$$
\begin{aligned}
\mathbf{g}_{S}= & -f\left(d T^{2}-2 \Psi d T d R+\Psi^{2} d R^{2}\right)+\frac{H^{2}}{f} d R^{2} \\
& +\tilde{r}^{2}\left(d \theta^{2}+\sin ^{2} \theta d \phi^{2}\right), \\
\widetilde{\mathbf{h}}= & -\frac{4 \epsilon m_{2}}{b^{3}} f \tilde{r}^{3}(d T-\Psi d R)[\cos \theta \sin \phi d \theta \\
& +\sin \theta \cos (2 \theta) \cos \phi d \phi]+\frac{m_{2}}{b^{3}} \tilde{r}^{2}\left(3 \sin ^{2} \theta \cos ^{2} \phi-1\right) \\
& \times\left[f^{2}\left(d T^{2}-2 \Psi d T d R+\Psi^{2} d R^{2}\right)\right. \\
& \left.+H^{2} d R^{2}+\left(\tilde{r}^{2}-2 m_{1}^{2}\right)\left(d \theta^{2}+\sin ^{2} \theta d \phi^{2}\right)\right]
\end{aligned}
$$

where

$$
\begin{aligned}
\Psi(R)= & 2 m_{1}\left[R^{-1}\left(1-\frac{2 m_{1}}{R}\right)^{-1} \psi(R)\right. \\
& \left.+\psi^{\prime}(R) \ln \left(\frac{R}{2 m_{1}}-1\right)\right], \\
H(R)= & 1+m_{1}\left(1+\frac{m_{1}}{4 R}\right) \eta^{\prime}(R)-\frac{m_{1}^{2}}{4 R^{2}} \eta(R), \\
f(R)= & 1-\frac{2 m_{1}}{\tilde{r}(R)},
\end{aligned}
$$

and $\tilde{r}$ is given in terms of $R$ in Eq. (4). Since the linearized Einstein equation is a tensor equation [see, e.g., Eq. (7.5.15) in [3]] and we have only performed a coordinate transformation, $\widetilde{\mathbf{h}}$ remains a solution to this equation on the background $\mathbf{g}_{S}$. However, the $\phi$ dependence of the perturbation $\widetilde{\mathbf{h}}$ does not correspond to the second black hole's rotating tidal field-we set $\Omega=0$ above. To remedy this, we simply change $\phi$ to $\phi-\Omega T$ in the components of $\widetilde{\mathbf{h}}$, which yields a new perturbation $\mathbf{h}$. Note that this replacement is not a coordinate transformation; a new tensor $\mathbf{h}$ is defined. Also note that $T$ becomes an ingoing coordinate near the horizon, so the time dependence $\phi-\Omega T$ causes no problems on the horizon. This simple remedy works for the following reason: if we solve the linearized Einstein equation order by order in $\epsilon$, then time derivatives of the components of $\mathbf{h}$ produce factors of $m_{1} \Omega \sim \boldsymbol{\epsilon}^{3}$ and can thus be neglected. The perturbation $\mathbf{h}$ is given by

$$
\begin{aligned}
\mathbf{h}= & -\frac{4 \epsilon m_{2}}{b^{3}} f \tilde{r}^{3}(d T-\Psi d R)[\cos \theta \sin (\phi-\Omega T) d \theta \\
& +\sin \theta \cos (2 \theta) \cos (\phi-\Omega T) d \phi] \\
& +\frac{m_{2}}{b^{3}} \tilde{r}^{2}\left[3 \sin ^{2} \theta \cos ^{2}(\phi-\Omega T)-1\right] \\
& \times\left[f^{2}\left(d T^{2}-2 \Psi d T d R+\Psi^{2} d R^{2}\right)+H^{2} d R^{2}+\left(\tilde{r}^{2}-2 m_{1}^{2}\right)\right. \\
& \left.\times\left(d \theta^{2}+\sin ^{2} \theta d \phi^{2}\right)\right] .
\end{aligned}
$$

We have now arrived at a metric $\mathbf{g}=\mathbf{g}_{S}+\mathbf{h}$, where $\mathbf{g}_{S}$ is given in Eq. (5) and $\mathbf{h}$ in Eq. (10), which is valid from the black hole's interior up, into, and through the buffer zone around the hole, and is written in coordinates that are well behaved throughout this region.

\section{TRANSFORMATION TO COROTATING COORDINATES}

The next step is to transform the metric $\mathbf{g}$ to corotating post-Newtonian coordinates $(t, x, y, z)$ using the transformation given in Eqs. (4.22) and (4.23) of [1]. This transformation contains a rotation that can be performed by first defining $\varphi=\phi-\Omega T$ and then setting $\Gamma=R \sin \theta \cos \varphi, \quad \Lambda$ $=R \sin \theta \sin \varphi$, and $Z=R \cos \theta$. To complete the transforma- 
tion, define the functions $P_{\alpha \beta}(x, y, z)$ for $\alpha, \beta=0, \ldots, 3$ to be components of the metric $\mathbf{g}$ in coordinates $(T, \Gamma, \Lambda, Z)$; write these components as functions of $(x, y, z)$ using Eqs. (4.22) and (4.23) in [1]. The functions $P_{\alpha \beta}$ are given by

$$
\begin{aligned}
& P_{00}=-f+\frac{\tilde{r}^{2}}{R^{2}} \Omega^{2}\left(\Gamma^{2}+\Lambda^{2}\right)-\frac{4 \epsilon m_{2} \tilde{r}^{3}}{b^{3} R^{3}}\left(2 Z^{2}-R^{2}\right) f \Omega \Gamma \\
& +\frac{m_{2} \tilde{r}^{2}}{b^{3} R^{2}}\left(3 \Gamma^{2}-R^{2}\right)\left[f^{2}+\frac{\Omega^{2}}{R^{2}}\left(\tilde{r}^{2}-2 m_{1}^{2}\right)\left(\Gamma^{2}+\Lambda^{2}\right)\right], \\
& P_{01}=P_{10}=\frac{\Gamma}{R} f \Psi-\frac{\tilde{r}^{2}}{R^{2}} \Omega \Lambda \\
& -\frac{2 \epsilon m_{2} \tilde{r}^{3}}{b^{3} R^{3}} f\left[\Gamma \Lambda-\frac{\Omega}{R} \Psi \Gamma^{2}\left(2 Z^{2}-R^{2}\right)\right] \\
& +\frac{m_{2} \tilde{r}^{2}}{b^{3} R^{2}}\left(3 \Gamma^{2}-R^{2}\right)\left[-\frac{\Gamma}{R} f^{2} \Psi-\frac{\Omega \Lambda}{R^{2}}\left(\tilde{r}^{2}-2 m_{1}^{2}\right)\right], \\
& P_{02}=P_{20}=\frac{\Lambda}{R} f \Psi+\frac{\tilde{r}^{2}}{R^{2}} \Omega \Gamma \\
& -\frac{2 \epsilon m_{2} \tilde{r}^{3}}{b^{3} R^{3}} f\left[Z^{2}-\Gamma^{2}-\frac{\Omega}{R} \Psi \Gamma \Lambda\left(2 Z^{2}-R^{2}\right)\right] \\
& +\frac{m_{2} \tilde{r}^{2}}{b^{3} R^{2}}\left(3 \Gamma^{2}-R^{2}\right)\left[-\frac{\Lambda}{R} f^{2} \Psi+\frac{\Omega \Gamma}{R^{2}}\left(\tilde{r}^{2}-2 m_{1}^{2}\right)\right], \\
& P_{03}=P_{30}=\frac{Z}{R} f \Psi-\frac{2 \epsilon m_{2} \tilde{r}^{3}}{b^{3} R^{3}} f\left[-\Lambda Z-\frac{\Omega}{R} \Psi \Gamma Z\left(2 Z^{2}-R^{2}\right)\right] \\
& +\frac{m_{2} \tilde{r}^{2}}{b^{3} R^{2}}\left(3 \Gamma^{2}-R^{2}\right)\left(-\frac{Z}{R} f^{2} \Psi\right), \\
& P_{11}=\frac{\Gamma^{2}}{R^{2}}\left(-f \Psi^{2}+\frac{H^{2}}{f}-\frac{\tilde{r}^{2}}{R^{2}}\right)+\frac{\tilde{r}^{2}}{R^{2}} \\
& +\frac{4 \epsilon m_{2} \tilde{r}^{3}}{b^{3} R^{4}} f \Psi \Gamma^{2} \Lambda \frac{m_{2} \tilde{r}^{2}}{b^{3} R^{2}}\left(3 \Gamma^{2}-R^{2}\right) \\
& \times\left[\frac{\Gamma^{2}}{R^{2}}\left(f^{2} \Psi^{2}+H^{2}\right)+R^{-2}\left(\tilde{r}^{2}-2 m_{1}^{2}\right)\left(1-\frac{\Gamma^{2}}{R^{2}}\right)\right], \\
& P_{22}=\frac{\Lambda^{2}}{R^{2}}\left(-f \Psi^{2}+\frac{H^{2}}{f}-\frac{\tilde{r}^{2}}{R^{2}}\right)+\frac{\tilde{r}^{2}}{R^{2}} \\
& +\frac{4 \epsilon m_{2} \tilde{r}^{3}}{b^{3} R^{4}} f \Psi \Lambda\left(Z^{2}-\Gamma^{2}\right)+\frac{m_{2} \tilde{r}^{2}}{b^{3} R^{2}}\left(3 \Gamma^{2}-R^{2}\right) \\
& \times\left[\frac{\Lambda^{2}}{R^{2}}\left(f^{2} \Psi^{2}+H^{2}\right)+R^{-2}\left(\tilde{r}^{2}-2 m_{1}^{2}\right)\left(1-\frac{\Lambda^{2}}{R^{2}}\right)\right],
\end{aligned}
$$

$$
\begin{aligned}
P_{33}= & \frac{Z^{2}}{R^{2}}\left(-f \Psi^{2}+\frac{H^{2}}{f}-\frac{\tilde{r}^{2}}{R^{2}}\right)+\frac{\tilde{r}^{2}}{R^{2}}-\frac{4 \epsilon m_{2} \tilde{r}^{3}}{b^{3} R^{4}} f \Psi Z^{2} \Lambda \\
& +\frac{m_{2} \tilde{r}^{2}}{b^{3} R^{2}}\left(3 \Gamma^{2}-R^{2}\right)\left[\frac{Z^{2}}{R^{2}}\left(f^{2} \Psi^{2}+H^{2}\right)\right. \\
& \left.+R^{-2}\left(\tilde{r}^{2}-2 m_{1}^{2}\right)\left(1-\frac{Z^{2}}{R^{2}}\right)\right], \\
P_{12}= & P_{21}=\frac{\Gamma \Lambda}{R^{2}}\left(-f \Psi^{2}+\frac{H^{2}}{f}-\frac{\tilde{r}^{2}}{R^{2}}\right) \\
& +\frac{2 \epsilon m_{2} \tilde{r}^{3}}{b^{3} R^{4}} f \Psi \Gamma\left(-\Gamma^{2}+\Lambda^{2}+Z^{2}\right)+\frac{m_{2} \tilde{r}^{2}}{b^{3} R^{4}}\left(3 \Gamma^{2}-R^{2}\right) \\
& \times\left[f^{2} \Psi^{2}+H^{2}-R^{-2}\left(\tilde{r}^{2}-2 m_{1}^{2}\right)\right] \Gamma \Lambda, \\
P_{13}= & P_{31}=\frac{\Gamma Z}{R^{2}}\left(-f \Psi^{2}+\frac{H^{2}}{f}-\frac{\tilde{r}^{2}}{R^{2}}\right)+\frac{m_{2} \tilde{r}^{2}}{b^{3} R^{4}}\left(3 \Gamma^{2}-R^{2}\right) \\
& \times\left[f^{2} \Psi^{2}+H^{2}-R^{-2}\left(\tilde{r}^{2}-2 m_{1}^{2}\right)\right] \Gamma Z, \\
P_{23}= & P_{32}=\frac{\Lambda Z}{R^{2}}\left(-f \Psi^{2}+\frac{H^{2}}{f}-\frac{\tilde{r}^{2}}{R^{2}}\right) \\
& +\frac{2 \epsilon m_{2} \tilde{r}^{3}}{b^{3} R^{4}} f \Psi Z\left(-\Gamma^{2}-\Lambda^{2}+Z^{2}\right)+\frac{m_{2} \tilde{r}^{2}}{b^{3} R^{4}}\left(3 \Gamma^{2}-R^{2}\right) \\
& \times\left[f^{2} \Psi^{2}+H^{2}-R^{-2}\left(\tilde{r}^{2}-2 m_{1}^{2}\right)\right] \Lambda Z, \\
&
\end{aligned}
$$

where $\epsilon=(m / b)^{1 / 2}, \Omega=\left(1-m_{1} m_{2} / m b\right)\left(m / b^{3}\right)^{1 / 2}, R=\left(\Gamma^{2}\right.$ $\left.+\Lambda^{2}+Z^{2}\right)^{1 / 2}$, and $\Gamma, \Lambda$, and $Z$ are to be expressed in terms of $(x, y, z)$ via Eqs. (4.22) and (4.23) in [1]. The functions $\tilde{r}(R), \Psi(R), H(R)$, and $f(R)$ are given in Eqs. (4), (7), (8), and (9).

The remainder of the coordinate transformation from black hole coordinates $(T, R, \theta, \phi)$ to corotating postNewtonian coordinates $(t, x, y, z)$ can be done exactly as in [1]. The final metric in region I (see Fig. 1 in [1]) is given by Eq. (4.27) of [1], but with $P_{\alpha \beta}$ taken from Eq. (11) above. Define $\bar{P}_{\alpha \beta}$ to be $P_{\alpha \beta}$ [as given in Eq. (11)] with $m_{1}$ and $m_{2}$ exchanged. Then the metric in region II (that is, near the second black hole; see Fig. 1 in [1]) is given by Eq. (4.28) in [1], but with $\bar{P}_{\alpha \beta}$ taken from here. Note that the final metric components are everywhere explicitly independent of time $t$.

To summarize, the expressions for the metric components given in Sec. V of [1] are valid in ingoing coordinates if $P_{\alpha \beta}$ (and $\bar{P}_{\alpha \beta}$ ) are taken from Eq. (11) and the functions $\tilde{r}(R)$, $\Psi(R), H(R)$, and $f(R)$ are taken from Eqs. (4), (7), (8), and (9) with $\psi(R)$ and $\eta(R)$ having properties (i)-(iii) given below Eq. (4). In order to show explicitly that these components are nonsingular at the black holes' horizons, I write out $P_{\alpha \beta}$ for $R \leqslant \zeta_{1} m_{1}$. In this region, $\psi=1, \Psi(R)$ 
$=2 m_{1} R^{-1}\left(1-2 m_{1} / R\right)^{-1}, \eta=0, H=1, \tilde{r}=R$, and $f(R)=1$

$-2 m_{1} / R$. Therefore, for $R \leqslant \zeta_{1} m_{1}, P_{\alpha \beta}$ are given by

$$
\begin{aligned}
& P_{00}=-1+\frac{2 m_{1}}{R}+\Omega^{2}\left(\Gamma^{2}+\Lambda^{2}\right)-\frac{4 \epsilon m_{2}}{b^{3}}\left(1-\frac{2 m_{1}}{R}\right) \\
& \times\left(2 Z^{2}-R^{2}\right) \Omega \Gamma+\frac{m_{2}}{b^{3}}\left(3 \Gamma^{2}-R^{2}\right)\left[\left(1-\frac{2 m_{1}}{R}\right)^{2}\right. \\
& \left.+\Omega^{2}\left(1-\frac{2 m_{1}^{2}}{R^{2}}\right)\left(\Gamma^{2}+\Lambda^{2}\right)\right] \\
& P_{01}=P_{10}=\frac{2 m_{1}}{R^{2}} \Gamma-\Omega \Lambda-\frac{2 \epsilon m_{2}}{b^{3}}\left[\left(1-\frac{2 m_{1}}{R}\right) \Gamma \Lambda\right. \\
& \left.-\frac{2 m_{1}}{R^{2}} \Omega \Gamma^{2}\left(2 Z^{2}-R^{2}\right)\right] \\
& -\frac{m_{2}}{b^{3}}\left(3 \Gamma^{2}-R^{2}\right)\left[\frac{2 m_{1}}{R^{2}}\left(1-\frac{2 m_{1}}{R}\right) \Gamma\right. \\
& \left.+\Omega \Lambda\left(1-\frac{2 m_{1}^{2}}{R^{2}}\right)\right] \\
& P_{02}=P_{20}=\frac{2 m_{1}}{R^{2}} \Lambda+\Omega \Gamma-\frac{2 \epsilon m_{2}}{b^{3}}\left[\left(1-\frac{2 m_{1}}{R}\right)\left(Z^{2}-\Gamma^{2}\right)\right. \\
& \left.-\frac{2 m_{1}}{R^{2}} \Omega \Gamma \Lambda\left(2 Z^{2}-R^{2}\right)\right] \\
& -\frac{m_{2}}{b^{3}}\left(3 \Gamma^{2}-R^{2}\right)\left[\frac{2 m_{1}}{R^{2}}\left(1-\frac{2 m_{1}}{R}\right) \Lambda\right. \\
& \left.-\Omega \Gamma\left(1-\frac{2 m_{1}^{2}}{R^{2}}\right)\right] \\
& P_{03}=P_{30}=\frac{2 m_{1}}{R^{2}} Z+\frac{2 \epsilon m_{2}}{b^{3}}\left[\left(1-\frac{2 m_{1}}{R}\right) \Lambda Z\right. \\
& \left.+\frac{2 m_{1}}{R^{2}} \Omega \Gamma Z\left(2 Z^{2}-R^{2}\right)\right] \\
& -\frac{2 m_{1} m_{2}}{b^{3} R^{2}}\left(3 \Gamma^{2}-R^{2}\right)\left(1-\frac{2 m_{1}}{R}\right) Z, \\
& P_{11}=1+\frac{2 m_{1} \Gamma^{2}}{R^{3}}+\frac{8 \epsilon m_{1} m_{2}}{b^{3} R^{2}} \Gamma^{2} \Lambda \\
& +\frac{m_{2}}{b^{3}}\left(3 \Gamma^{2}-R^{2}\right)\left[1-\frac{2 m_{1}^{2}}{R^{2}}\left(1-\frac{3 \Gamma^{2}}{R^{2}}\right)\right],
\end{aligned}
$$

$$
P_{22}=1+\frac{2 m_{1} \Lambda^{2}}{R^{3}}+\frac{8 \epsilon m_{1} m_{2}}{b^{3} R^{2}} \Lambda\left(Z^{2}-\Gamma^{2}\right)+\frac{m_{2}}{b^{3}}\left(3 \Gamma^{2}\right.
$$$$
\left.-R^{2}\right)\left[1-\frac{2 m_{1}^{2}}{R^{2}}\left(1-\frac{3 \Lambda^{2}}{R^{2}}\right)\right]
$$$$
P_{33}=1+\frac{2 m_{1} Z^{2}}{R^{3}}-\frac{8 \epsilon m_{1} m_{2}}{b^{3} R^{2}} Z^{2} \Lambda
$$$$
+\frac{m_{2}}{b^{3}}\left(3 \Gamma^{2}-R^{2}\right)\left[1-\frac{2 m_{1}^{2}}{R^{2}}\left(1-\frac{3 Z^{2}}{R^{2}}\right)\right]
$$$$
P_{12}=P_{21}=\frac{2 m_{1} \Gamma \Lambda}{R^{3}}+\frac{4 \epsilon m_{1} m_{2}}{b^{3} R^{2}} \Gamma\left(R^{2}-2 \Gamma^{2}\right)
$$$$
+\frac{6 m_{1}^{2} m_{2}}{b^{3} R^{4}}\left(3 \Gamma^{2}-R^{2}\right) \Gamma \Lambda
$$$$
P_{13}=P_{31}=\frac{2 m_{1} \Gamma Z}{R^{3}}+\frac{6 m_{1}^{2} m_{2}}{b^{3} R^{4}}\left(3 \Gamma^{2}-R^{2}\right) \Gamma Z,
$$$$
P_{23}=P_{32}=\frac{2 m_{1} \Lambda Z}{R^{3}}+\frac{4 \epsilon m_{1} m_{2}}{b^{3} R^{2}} Z\left(2 Z^{2}-R^{2}\right)
$$$$
+\frac{6 m_{1}^{2} m_{2}}{b^{3} R^{4}}\left(3 \Gamma^{2}-R^{2}\right) \Lambda Z,
$$

where $\epsilon=(m / b)^{1 / 2}, \Omega=\left(1-m_{1} m_{2} / m b\right)\left(m / b^{3}\right)^{1 / 2}, R=\left(\Gamma^{2}\right.$ $\left.+\Lambda^{2}+Z^{2}\right)^{1 / 2}$, and $\Gamma, \Lambda$, and $Z$ are to be expressed in terms of $(x, y, z)$ via Eqs. (4.22) and (4.23) in [1]. Note that the quantities $P_{\alpha \beta}$ in Eq. (12) are all finite at the horizon $R$ $=2 m_{1}$.

\section{ACKNOWLEDGMENTS}

I thank Lee Lindblom, Mark Scheel, Kip Thorne, and Michele Vallisneri for useful discussions. This research was supported in part by NSF grant PHY-9900776.
[1] K. Alvi, Phys. Rev. D 61, 124013 (2000).

[2] L. Lehner, Class. Quantum Grav. 18, R25 (2001).
[3] R. M. Wald, General Relativity (University of Chicago Press, Chicago, 1984). 